\title{
Implicaciones de la acción educativa en espacios específicos de proyección profesional en España
}

\author{
Francisco Javier Blanco Encomienda \\ María José Latorre Medina \\ Universidad de Granada
}

\section{Resumen}

En los últimos años, ha aumentado considerablemente el número de publicaciones que ilustran actuaciones educativas en espacios excepcionales de proyección profesional, como aquellas que necesitan los menores enfermos y/u hospitalizados, no sólo desde el punto de vista físico o médico, sino también desde el psicológico y educativo. Concretamente, como parte del abordaje integral de las situaciones de atención a necesidades educativas especiales en los hospitales, surgen las aulas hospitalarias; entornos de enseñanza-aprendizaje que, dadas sus características peculiares de espacio y ubicación, demandan una intervención psicopedagógica eficaz, diferente de la ordinaria. El presente trabajo pretende ser una contribución en esta dirección, centrando su atención, primero, en descifrar el sentido de la Psicopedagogía en el contexto hospitalario. A este respecto, la literatura deja constancia de que la intervención psicopedagógica en las aulas hospitalarias cobra sentido e importancia al tener, como objetivo último, ayudar a reforzar y apoyar psicológica y educativamente los procesos de enfermedad y hospitalización infantil, con el fin de conseguir la mayor normalización en la vida del paciente y su familia. A continuación, el artículo profundiza en el estudio de una formación adecuada para el profesional de la Psicopedagogía Hospitalaria, identificando cuatro clusters de competencias: competencias pedagógico-didácticas, competencias político-institucionales, competencias interactivas y competencias específicas. Por último, recoge las principales implicaciones educativas del trabajo en este contexto, perfilando propuestas para la acción.

\section{Palabras clave}

Correo:

María José Latorre Medina

Facultad de Educación y

Humanidades de Ceuta

Universidad de Granada

C/ El Greco, $\mathbf{n}^{0} 10$

51002 CEUTA (ESPAÑA)

34657306434

E-mail: mjlator@ugr.es
Implicaciones educativas - Aulas hospitalarias - Intervención psicopedagógica - Perfil profesional. 


\title{
Implications of the educative action in spaces of specific professional orientation in Spain
}

\author{
Francisco Javier Blanco Encomienda \\ María José Latorre Medina \\ University of Granada
}

Contact:

María José Latorre Medina

Facultad de Educación y

Humanidades de Ceuta

Universidad de Granada

C/ El Greco, $n^{\circ} 10$

51002 CEUTA (ESPAÑA)

34657306434

E-mail: mjlator@ugr.es

\begin{abstract}
In the last years, there has been considerable increase in the number of publications depicting educative actions in special spaces of professional orientation, such as those required by sick and/or hospitalized youngsters, not just from the physical or medical viewpoint, but also from the psychological and educative one. In concrete terms, as part of the integral approach to situations of attention to the special educative needs in hospitals, emerge the 'hospital classes', teaching-learning environments which, given their specific characteristics of space and location, require an efficient psychopedagogical intervention, different from the normal one. The present work aims to contribute along these lines, centering its attention, firstly, at deciphering the meaning of Psychopedagogy within the hospital context. In this respect, the literature shows evidence of the fact that the psychopedagogical intervention in the hospital classes acquires significance and importance by having as its ultimate goal to help sustaining and supporting psychologically and educationally the children's processes of illness and hospitalization, with the purpose of preserving the best possible normality for the patient's life and those of their relatives. Next, the article goes further into the investigation of what would constitute an appropriate formation for the professional of Hospital Psychopedagogy, identifying four clusters of competences: pedagogical-didactical competences, political-institutional competences, interactive competences, and specific competences. Lastly, the text collects the main educative implications of the work in this context, lining up proposals for action.
\end{abstract}

\section{Keywords}

Educative implications - Hospital classes - Psychopedagogical intervention - Professional profile. 


\section{Introducción}

En los últimos años, ha aumentado considerablemente el número de publicaciones que refieren la necesidad de potenciar la atención integral a los menores enfermos y hospitalizados, no sólo desde el punto de vista físico o médico, sino también desde el psicológico y educativo (DE MANUELES, ORTIZ, SERRADAS, ESTALAYO, 2002). Es incuestionable que el niño enfermo y/u hospitalizado tiene, al igual que el niño sano, unas necesidades básicas que desarrollar, propias de cada etapa evolutiva y, por ello, derecho a la educación, en concreto, a la disponibilidad de educadores y medios que guien su proceso de aprendizaje dentro del contexto hospitalario, favorezcan la adaptación a este medio y le permitan expresar sus temores y ansiedades.

Como parte del ansiado abordaje integral de las situaciones de atención a necesidades educativas especiales (GRAU, 2003) en estos espacios excepcionales de proyección profesional surgen las aulas hospitalarias; lugares que, a su vez, demandan una intervención psicopedagógica eficaz como solución bastante apropiada a muchos casos particulares de menores en situaciones de especial fragilidad (BARCELÓ; LAMARCA, 2006), como son, en este caso, los niños enfermos y/u hospitalizados. Según Ortiz (1998), estos centros de enseñanza dentro de los hospitales se crearon para atender el derecho que todo menor tiene a recibir una educación general, incluso cuando sea necesaria su hospitalización. Y dadas sus características especiales de espacio y ubicación, así como por el tipo de alumnado que reciben con cualquier problema de salud, son aulas que requieren un tratamiento especial y una actuación psicoeducativa diferenciada respecto a la ordinaria.

El presente trabajo pretende ser una contribución en esta dirección, centrando su atención, primero, en descifrar el sentido de la Psicopedagogía en el contexto hospitalario. Dada su importancia, profundiza a continuación en el estudio de una formación adecuada para el profesional de la psicopedagogía hospitalaria, identificando competencias profesionales. Por último, recoge las principales implicaciones educativas de una intervención psicopedagógica eficaz en los hospitales, perfilando propuestas para la práctica.

\section{Sentido de la psicopedagogía en el contexto hospitalario}

De entrada, siguiendo a González-Simancas y Polaino-Lorente (1990), se puede concebir la Psicopedagogía Hospitalaria como íntimamente relacionada con la Pedagogía Social, pues dicha rama de la educación se ocupa, a nivel general, del estudio de la teoría y la práctica de la educación individualizada de grupos en situaciones excepcionales de aprendizaje, como son los sujetos enfermos y hospitalizados.

Más concretamente, Deasy (1993) matiza que la Psicopedagogía, en el contexto sanitario, debe entenderse como un servicio más ofertado por los hospitales, un espacio institucional desde el que se articulen las funciones de apoyo y ayuda psicológica, educativa y social al paciente pediátrico y su familia. Una oferta adaptada y diversificada a la edad y estado de salud de los enfermos para que cada paciente pueda participar en el proceso de enseñanza/aprendizaje más adecuado a sus circunstancias particulares y que, en general, pueda ser guiado con actuaciones que tiendan a mejorar su calidad de vida durante la enfermedad y la hospitalización.

Para López y Fernández (2006) cobra sentido e importancia la intervención psicopedagógica en aulas hospitalarias al poseer, como objetivo último, el ayudar a reforzar y apoyar psicológica y educativamente los procesos de enfermedad y hospitalización 
infantil, con el fin de conseguir la mayor normalización en la vida del paciente y su familia. Para los autores, la finalidad de la Psicopedagogía Hospitalaria es la misma que la de la educación, es decir, la búsqueda del desarrollo integral de la persona, persiguiendo la máxima evolución de todas sus capacidades, incluso en una situación anómala como es el hecho de estar enfermo y hospitalizado.

Con un planteamiento similar, Lizasoáin (2003) sostiene que esta finalidad global se consigue a través de un objetivo concreto: prevenir y evitar la marginación del proceso educativo de los niños en edad escolar que se encuentran ingresados en un hospital.

Pese a que son numerosos los objetivos asignados a este campo concreto de actuación profesional, éstos pueden sintetizarse en cuatro: el desarrollo de los aspectos cognitivos e intelectuales; el desarrollo motriz; el desarrollo afectivo; y el desarrollo social del paciente pediátrico.

\section{Campo de actuación de la Psi- copedagogía Hospitalaria}

Aunque la Psicopedagogía Hospitalaria hace extensible su intervención a las consultas ambulatorias y los hospitales de día, su ámbito propio de actuación es el centro hospitalario. Dentro de él, la tarea psicodidáctica se realiza fundamentalmente en el aula hospitalaria y en las habitaciones de los niños, cuando éstos no pueden acudir al aula.

En otro de los trabajos informados por Ortiz (1998) se recoge que los niños hospitalizados, al igual que el resto de escolares, tienen el mismo derecho a recibir una adecuada atención educativa en las aulas de apoyo que existen en los hospitales (aulas hospitalarias) que, por responder a las necesidades específicas que presentan los niños enfermos, son consideradas como 'aulas inclusivas' (CABALLERO, 2007; LEÓN, 2001). En los hospitales, estas aulas se establecen como áreas abiertas y flexibles, donde el paciente pediátrico puede acudir libremente, ausentándose siempre que lo requiera su asistencia sanitaria.

Normalmente ubicadas en la planta de pediatría, González (2004) dibuja estos espacios como habitaciones amplias, alegres, bien ventiladas y luminosas, sumamente atractivas, llenas de colores, libros, juegos, material docente etc. Desde el punto de vista de los familiares y profesionales, las aulas hospitalarias son espacios físicos absolutamente necesarios en los hospitales para el bienestar de los niños enfermos. Por ello, deben ser un lugar seguro y normalizador para estos alumnos, donde, además de la función educativa, vean o tengan para ellos un significado relacional, ocupacional e integrador. Tal como se desprende del estudio emprendido por Barrueco, Ortiz, Calvo, De Manueles y Martín (1997), las aulas hospitalarias son clave para darle contenido educativo a los ratos de ocio, para recuperar el retraso académico y, sobre todo, como vía de enriquecimiento personal y social.

Parece ser que, en nuestro país, la Psicopedagogía Hospitalaria ha comenzado su andadura centrándose en la atención a los niños hospitalizados y sus familias, básicamente en tres direcciones: la adaptación del niño y su familia al hospital, la evitación del desarraigo infantil con respecto a su medio habitual (hogar, escuela, amigos, ciudad, etc.) y el aporte de medios psicopedagógicos adecuados para ayudar al paciente y sus progenitores a que sean capaces de autorregular y dirigir sus conductas y emociones en las situaciones de dificultad añadida de enfermedad y hospitalización.

Siguiendo a Grau y Ortiz (2001), tales funciones se deberían realizar en el hospital, pero en constante colaboración con la 
escuela, para darles operatividad y funcionalidad educativa, ya que lo que se persigue es la reinserción integral del paciente pediátrico en su entorno formativo ordinario.

\section{Principios orientadores y organizativos de la atención psicopedagógica en aulas hospitalarias}

Grau (2001) resume en cinco los principios orientadores de la atención a las necesidades educativas especiales de los niños hospitalizados:

a. Se realiza por un docente en las unidades escolares de apoyo o aulas hospitalarias y tiene como funciones básicas: compensar el posible retraso académico; ocupar el tiempo libre; promover la independencia y confianza del niño en el medio hospitalario; compartir las preocupaciones y los problemas de los niños, animándoles para que tengan una actitud positiva hacia la enfermedad; mejorar su estabilidad psíquica ante situaciones delicadas referidas a la ansiedad y al miedo ante determinadas pruebas por las que tienen que pasar; mejorar su estabilidad en lo que se refiere a la inquietud por conocer si se van a curar pronto, al desconocimiento del tiempo de ingreso y a la preocupación, sobre todo, por encontrarse solos.

b. Estará coordinada con los centros educativos de referencia y con los servicios de atención educativa domiciliaria, si el niño los necesitara, ya que el objetivo primordial es que el niño se incorpore de nuevo al colegio con las máximas garantías de éxito y pueda desarrollar, en la medida de sus posibilidades, una vida lo más normalizada posible.

c. Debe atender a la familia de los niños enfermos $\mathrm{y} / \mathrm{u}$ hospitalizados a través de la creación de unidades de orientación familiar. d. Se adaptará a las fases de hospitalización del niño (ingreso, estancia y alta). En el ingreso, se intentará hacer más familiar el medio hospitalario, tratando de eliminar la incertidumbre y el miedo al pronóstico de la enfermedad. Durante la estancia, se preparará al niño para todas las pruebas diagnósticas e intervenciones terapéuticas necesarias, y se tratarán los problemas emocionales, intentando continuar la actividad escolar. Finalmente, en el alta, habrá que preparar al niño para enfrentarse de nuevo a su entorno familiar, escolar y social, pudiendo llevar a cabo una vida lo más normalizada posible.

e. Se centrará fundamentalmente en una visión global de la superación de la enfermedad, que tenga en cuenta la reeducación física, emocional y cognitiva, mediante la utilización de técnicas creativas y de expresión, entre otras.

Respecto a los principios organizativos, la actuación psicoeducativa que se lleve a cabo debe ser un eslabón dentro de un sistema que permita al niño enfermo recibir una atención adaptada a sus necesidades para que pueda desarrollar una vida activa, a pesar de su enfermedad, de sus secuelas y de los tratamientos recibidos. Además, la atención prestada debe ser parte integral de los programas de tratamiento médico y una responsabilidad y un trabajo compartido entre los padres y el equipo interdisciplinario de profesionales que atienden al niño (médicos, enfermeros, psicopedagogos, docentes, pedagogos, asistentes sociales, etc.), dado que la continuidad escolar del niño -escuela, familia y hospital- transmite un mensaje de esperanza en su futuro, además de permitirle desarrollar al máximo sus habilidades sociales y cognitivas.

En relación con ello, Castillo (2005), Grau (2003) y Ullán (2005) subrayan que la respuesta educativa a las necesidades educativas especiales, derivadas de la enfermedad 
del niño, implica: la colaboración de equipos interdisciplinarios, tanto en el ámbito hospitalario como en el familiar y escolar; la atención a los problemas emocionales del niño y de la familia; el desarrollo de estrategias de adaptación a la enfermedad y al contexto hospitalario, y la elaboración de adaptaciones curriculares que intenten paliar, lo más pronto posible, las secuelas derivadas de la enfermedad que afectan a la capacidad de aprendizaje del niño. Por todo ello, se hace imprescindible la existencia de un programa hospital/escuela bien definido y organizado, que incluya: las aulas hospitalarias, la atención educativa domiciliaria y los programas de preparación para la vuelta al colegio.

\section{Marco legislativo español de las actuaciones educativas en hospitales}

La actividad legislativa española sobre la actuación en el medio hospitalario es paralela a la europea, en lo que respecta a las actividades psicopedagógicas que se deben desarrollar en los hospitales con los niños ingresados. Se recogen en la Carta Europea de Derechos del Niño Hospitalizado, aprobada por el Parlamento Europeo en $1986^{1}$.

Haciendo un excelente repaso de la legislación española sobre las actuaciones educativas en centros hospitalarios, Castillo (2006a) y Grau (2001) esgrimen en sus estudios que si bien la Ley 13/1982, de 7 de abril, sobre la Integración Social de los Minusválidos establece en su artículo 29 la existencia de las aulas hospitalarias (una sección pedagógica) en todos los hospitales públicos y privados con subvención de fondos públicos, habrá que esperar al Real Decreto 334/1985, de 6 de marzo, de Ordenación de la Educación Especial, para que se dote a los hospitales de profesores y se creen las aulas hospitalarias.

1. http://www.cnice.mecd.es/proyectos/aulashosp/derechos.htm
Se suscriben convenios en las comunidades autónomas entre la administración educativa y la sanitaria, de forma que el profesorado de estas aulas será de la administración educativa, mientras que la sanitaria proporcionará los espacios físicos y la financiación para la dotación de dichas aulas.

En la década de los noventa, la Ley Orgánica General del Sistema Educativo (LOGSE) hace referencia a las escuelas en los hospitales. En 1991 se elabora el documento que lleva por título 'Aulas Hospitalarias' y se crea el Programa de Educación Compensatoria para las Aulas Hospitalarias. Los Reales Decretos que desarrolla la LOGSE en materia de alumnos con necesidades educativas especiales (Real Decreto 696/1995, de 28 de abril, de Ordenación de la educación de los alumnos con necesidades educativas especiales, y el Real Decreto 299/1996, de 28 de febrero, de Ordenación de las acciones dirigidas a la compensación de las desigualdades en educación) establecen la creación de unidades escolares de apoyo en los centros hospitalarios mantenidos con fondos públicos.

A este respecto, merecen especial mención los objetivos recogidos en las directrices que el Ministerio de Educación y Cultura y la Subdirección General de Educación Compensatoria dictaminaron en su día en el denominado Programa de Aulas Hospitalarias (Guillén y Mejía, 2002). Estos objetivos son:

- Pedagógicos. Se pretende evitar la marginación del proceso educativo y posibilitar así su incorporación a la vida escolar normal, una vez superada la enfermedad.

- Psicológicos. Se pretende conseguir la mejor adaptación posible de los niños al nuevo medio, así como que lleguen a comprender qué es lo que les está sucediendo y por qué. 
- Sociales. Se pretende crear un ambiente lo más cercano a su vida diaria, satisfaciendo la necesidad de relacionarse que todo niño en edad escolar-necesita.

Años después, el convenio suscrito el 18 de mayo de 1998 entre los Ministerios de Educación y Cultura y de Sanidad y Consumo y el Instituto Nacional de Salud (INSALUD), para la atención educativa de los niños hospitalizados, aprueba la creación del aula hospitalaria en el servicio de pediatría con la inclusión de la más alta tecnología.

Por esta época, también las Comunidades Autónomas de Andalucía, Canarias, Cataluña, Galicia y la Comunidad Valenciana aprueban disposiciones con carácter genérico que establecen las aulas hospitalarias o unidades escolares de apoyo en los hospitales, aunque solamente la Comunidad de Madrid y el País Vasco elaboraron una normativa específica sobre el funcionamiento de dichas aulas.

\section{Preparación de profesionales de la Psicopedagogía Hospitalaria: competencias y} formación

Son muchos los profesionales involucrados en las actividades de Psicopedagogía Hospitalaria, fundamentalmente psicopedagogos, pedagogos, psicólogos educativos y maestros, cada uno de los cuales necesita formación para trabajar con garantías de calidad en esos espacios no nuevos de creación pero sí de proyección profesional. Este apartado centra su atención en la formación específica que necesitan los profesionales de la Psicopedagogía Hospitalaria, identificando competencias específicas.

En primer lugar, para el establecimiento de las competencias personales y profesionales específicas, se ha adaptado el modelo de Braslavsky (1998), dando lugar a cuatro de las cinco competencias señaladas. De este modo, los profesionales de la Psicopedagogía Hospitalaria que trabajen actualmente, tanto dentro del centro hospitalario como a nivel de domicilio, deberán poseer las siguientes competencias: competencias vinculadas, sobre todo, a la resolución de los problemas o desafíos más coyunturales, a las que denomina pedagógico-didáctica y político-institucional; competencias vinculadas a desafíos más estructurales y de unión de sinergias, llamadas interactivas y competencias vinculadas con los necesarios procesos de especialización y orientación de su práctica profesional, denominadas especificadoras.

A continuación, se desglosan estas competencias específicas del profesional de la Psicopedagogía Hospitalaria, en su doble vertiente (profesional hospitalario y profesional de atención domiciliaria). No obstante, hay que tener en cuenta que la labor del profesional domiciliario se centra más en la normalización de la vida del niño desde el punto de vista académico, esto es, de continuación con el currículo escolar y recuperación de posibles lagunas en el aprendizaje, mientras que el profesional hospitalario centra más su objetivo de normalización de la vida del niño en el propio hospital a través de la desdramatización de este contexto -de por sí agresivo- mediante actividades no sólo de tipo académico. Por otra parte, el primero trabaja con un único alumno, mientras que el segundo lo hace con un grupo heterogéneo. Sin embargo, y aunque varíe el número de alumnos con los que interactúan en un momento determinado, el trabajo en ambos casos debe ser individualizado.

Las competencias específicas que se han identificado anteriormente se resumen en los siguientes cuadros: 


\section{Cuadro 1.}

\section{Competencias pedagógico-didácticas}

Son facilitadoras de procesos de aprendizaje cada vez más autónomos. Los profesionales deben conocer, saber seleccionar, utilizar, evaluar, perfeccionar y recrear 0 crear estrategias de intervención didáctica efectivas. Esto incluye a las nuevas tecnologías de la información y la comunicación.

Capacidades, destrezas y actitudes con relación a:

- Recuperación de lagunas en el aprendizaje.

- Ayuda en la continuidad de los estudios.

- Ayuda en la promoción escolar.

- Mejora de los potenciales de aprendizaje.

- Trabajo (normalmente el número de horas reales de dedicación son mayores que las un maestro).

- Expresar respeto y comprensión hacia la enfermedad.

- La autoformación.

- Fortaleza emocional y personalidad equilibrada.

- Enfrentarse y actuar originalmente ante el dolor ajeno.

- Adquisición de estrategias y recursos psicopedagógicos que le posibiliten una respuesta lo más adecuada posible a las características de cada sujeto concreto.

- Solucionar problemas (organizar los tiempos).

- Manejar como recursos educativos las diversas experiencias que trae cada alumno.

- Mantener un registro individual que incluya los datos más importantes de su historial escolar y médico.

- Saber hacer adaptaciones curriculares individualizadas y actividades de refuerzo educativo.

- Conocimientos en Nuevas Tecnologías de la Información y la Comunicación.

- Creatividad e imaginación.

- Adaptación y empatía.

- Iniciativa y optimismo (animar a los niños a que tengan una actitud positiva ante su enfermedad).

- Comunicación.

- Generar actitudes de autoridad moral para despertar confianza y seguridad en el alumno y su familia.

- Actividades lúdico-formativas.

- Técnicas de animación grupal.

- Manejo simultáneo e individualizado de grupos heterogéneos (edad, patología, necesidades).

- Observar las conductas y situaciones del niño.

- Buscar, diseñar e implementar respuestas a corto, medio o largo plazo.

- Motivar al alumno para que participe activamente en las actividades del aula hospitalaria. 


\section{Cuadro 2.}

\section{Competencias político-institucionales}

Hacen referencia a la capacidad de articular lo macro con lo micro: lo que se dispone en el sistema educativo con lo que es necesario programar, llevar adelante y evaluar al nivel de aula hospitalaria 0 domicilio particular.

Capacidades, destrezas y actitudes relacionadas con:

- Conocimiento del currículo oficial de cada materia, en los diferentes niveles, así como del programa del centro de referencia de cada alumno.

- Coordinación de su trabajo diario con el programa de educación del centro de referencia, sobre todo para aquellos casos de largas hospitalizaciones, mediante la expresión clara de objetivos y procedimientos de aprendizaje, con el fin de evitar la confusión en el niño.

- Flexibilidad curricular.

- Adaptación a los diferentes contextos.

Cuadro 3.

\section{Competencias interactivas}

La especificidad de sus acciones y el hecho de compartir un espacio y unos recursos limitados obligan al profesional de la Psicopedagogía Hospitalaria (que ejerce en el hospital 0 a nivel de domicilio) a ser especialista en unión de las sinergias. Deben saber coordinarse con todos los profesionales implicados en la mejora de la calidad de vida de los niños y jóvenes enfermos.

Capacidades, destrezas y actitudes con relación a:

- Considerar, de forma sistemática, al niño, la familia, el personal sanitario y trabajar con todos ellos la nueva situación.

- Trabajo cooperativo e interprofesional.

- Flexibilidad y adaptabilidad al trabajo de otros.

- Negociación.

- Comunicación.

- Adecuada organización y coordinación, planificadas no sólo a nivel interno del propio centro hospitalario

o domicilio, sino también a nivel externo. Deben ser expertos en coordinación interna y en coordinación externa. 


\section{Competencias especificadoras}

\section{Es un mayor dominio de contenidos referidos al campo concreto de la atención educativa a niños y jóvenes enfermos y/u hospitalizados.}

Capacidades, destrezas y actitudes relacionadas con:

- Conocimiento de terminología médica.

- Conocimiento de las características psicosociológicas del niño y/o adolescente enfermo según las diferentes etapas evolutivas.

- Diagnóstico de los efectos de la enfermedad y de los tratamientos en el funcionamiento académico y cognitivo de los niños. Conocimientos profundos en diagnóstico psicopedagógico.

- Cómo informar a los alumnos, padres y otros profesores de las secuelas neurológicas, sensoriales, motrices y emocionales de la enfermedad y los tratamientos.

- Organización de un Hospital (funciones, características psicosociales y complejidad) y los recursos que ofrece desde un punto de vista educativo.

- Cómo vencer la resistencia del niño a volver a la escuela.

- Idea clara y firme de "dolor", "enfermedad" y "muerte".

- Afrontar situaciones difíciles (muerte).

- Aprender a "estar" de una manera educativa con niños enfermos, por medio del "diálogo y la compañía".

- Colaborar con el médico sin interferir con la función curativa que éste desempeña. Saber ocupar un segundo puesto, ya que la importancia de la función que realiza es subsidiaria y está subordinada a la del clínico. Estar dispuesto a sacrificar la eficacia de los rendimientos académicos a la mejor adaptación del niño al hospital (en el caso del profesional que trabaja en el centro hospitalario), a sacrificar los aprendizajes a la salud (sacrificar la programación curricular a la optimización de la evolución clínica, en el caso del profesional domiciliario).

Existe unanimidad entre los autores y profesionales en ejercicio al considerar que, para poder llevar a cabo una acción integral con el niño enfermo, con los padres y otros profesionales, la formación de los profesionales de la Psicopedagogía Hospitalaria ha de ir en la línea de las competencias descritas de cara a que el trabajo en las aulas hospitalarias y/o en los domicilios sea más eficaz, además de permitirles la utilización de estrategias pedagógicas más flexibles y el ingreso-reingreso a la 'formación por competencias', lo cual haría posible una formación inicial y un desarrollo profesional de mayor calidad.
Asumiendo lo anterior, los ejes formativos básicos podrían ser los siguientes: estructura y servicios hospitalarios; enfermedad en la infancia y repercusiones de la ausencia de salud en todos los ámbitos del paciente; diagnóstico psicopedagógico y de programas; acción tutorial y orientación; evaluación y seguimiento; realización de adaptaciones curriculares individualizadas; adaptaciones del entorno a las necesidades; realidad de la persona enferma para facilitar y promover su autonomía; trabajo cooperativo multiprofesional y multicéntrico; investigación en la acción, sin olvidar la referencia permanente de la escuela para todos 
(inclusiva y no marginadora); formación de la personalidad y el equilibrio emocional; tratamiento de las áreas curriculares en el contexto hospitalario, adecuando recursos, metodologías y actividades; y la práctica en las artes creativas.

Junto a esos contenidos teóricos-prácticos, se propone el aprendizaje de las nuevas tecnologías por su valor como medio de comunicación, de intercambio, de información, de adquisición de conocimientos (DOVAL, 2000); el diseño y elaboración de un proyecto curricular adaptado a las aulas hospitalarias; la gestión del aula y del servicio de atención domiciliaria (saber hacer informes, memorias de actividades, trabajar con bases de datos y hojas de cálculo, gestión económica etc.) y la elaboración y diseño de programas de atención domiciliaria.

Un elemento importante a destacar es que esos planes incorporaran créditos prácticos debido a la relevancia que tienen en cualquier programa de formación para la construcción de saberes profesionales (TONATTO; PROENÇA, 2007) y consolidación de competencias personales y profesionales.

Sea como fuere, la triste realidad es que en Europa hay más de 7500 profesionales de la Pedagogía y Psicopedagogía Hospitalaria en activo sin que existan ambas especialidades en ningún plan de formación universitario. Según Lizasoáin (2000), la mayoría de los profesionales europeos han recibido algún curso en educación especial, de manera que tras los tres o cuatro años de formación universitaria, el colectivo al que nos referimos cursa, en la mayoría de los casos, un año o dos de postgrado.

En España, la situación es aún peor, pues sólo unas pocas Universidades (Vigo, Salamanca, Valencia, Navarra, Complutense de Madrid) han incluido recientemente en sus programas formativos asignaturas, con carácter optativo, que abarcan específicamente esa temática, pero no son suficientes. Tampoco hay criterios comunes de diseño de la programación de la materia, ni tampoco masters o cursos de postgrado. Por otra parte, las experiencias de formación desarrolladas desde la
Administración Educativa (Centros de Formación Continuada del Profesorado, Direcciones Generales de Formación del Profesorado e Innovación Educativa etc.) son anecdóticas y no están diseñadas bajo la premisa de competencias a desarrollar en la línea de trabajo propugnada dentro del Espacio Europeo de Educación Superior (EEES).

\section{Implicaciones educativas de la intervención psicodidáctica en hospitales}

Los destinatarios principales de la intervención psicoeducativa desarrollada en entornos hospitalarios son, sin duda, los niños que, en edad escolar obligatoria, se encuentren hospitalizados o no puedan asistir a un centro de enseñanza por problemas de salud. Ortiz y Serradas (2002) sostienen que esta actuación psicopedagógica debe articularse desde el centro de referencia del alumno, con la intención de integrar y normalizar las necesidades formativas desde el centro escolar de origen y de acuerdo con el profesorado del mismo.

Las implicaciones educativas de la atención psicopedagógica en estos centros extraordinarios pueden clasificarse en dos grandes grupos: por un lado, actividades lúdicas, que son aquéllas que inciden en el desarrollo de la personalidad; y, por otro lado, actividades educativas, que son las que inciden en materias de aprendizaje escolar, según las condiciones de cada paciente pediátrico (PALOMO, 1992).

Las primeras actividades irían dirigidas a la contribución del desarrollo armónico de la personalidad y a la creación de una disposición positiva para la realización de las segundas, las actividades educativas, relacionadas con el proceso de enseñanza-aprendizaje del currículo escolar o su adaptación. Según González-Simancas y Polaino-Lorente (1990), estas actividades académicas pueden no estar presentes en el contexto hospitalario debido, por una parte, a que no constituyan el objetivo de la atención médica $\mathrm{y}$, por otra, a la imposibilidad de ser 
atendidas por el ya sobrecargado personal sanitario. Sin embargo, es necesaria la inclusión de estas actividades adaptadas al contexto donde se van a realizar, el hospital, de cara a cubrir las necesidades de instrucción que tiene el niño hospitalizado y, de este modo, favorecer la educación de los pacientes pediátricos en edad escolar.

De acuerdo con Barrueco, Ortiz, Calvo, de Manueles y Martín (1997), esas-actividades educativas no sólo estarán orientadas a la consecución de metas estrictamente académicas, sino que proyectarán también educar al niño en su enfermedad, la utilización efıcaz de su tiempo libre y el fomento, dentro de sus posibilidades, de continuar con una vida social y de relación lo más parecida a la que llevaba antes de su padecimiento (ALVES; ORTIZ, SERRADAS, 2004).

Para Castillo (2006a), nada más ingresar el niño en el hospital, el psicopedagogo hospitalario debería programar todas aquellas actividades encaminadas a conseguir los objetivos básicos que guían su actuación educativa en el hospital, así como aquellas otras actividades que ayuden a potenciar la creatividad del alumno enfermo, relacionadas con la intensificación de tareas de expresión artística, talleres etc., teniendo en cuenta que la realización de las mismas siempre dependerá de la disposición que demuestre el niño hospitalizado (de su concentración, autoestima, relajación, ánimo).

Desde el punto de vista psicológico y educativo, algunas actividades básicas que podrían llevarse a cabo son las siguientes (GONZÁLEZ-SIMANCAS; POLAINOLORENTE, 1990):

- Evaluación inicial del alumno.

- Formulación de objetivos generales e individualizaciones según las necesidades escolares de cada sujeto, efectos biopsíquicos de la enfermedad y factores psicosociales de la hospitalización.

- Actividades de enseñanza-aprendizaje más adecuadas a los objetivos.
- Evaluación periódica, asesoramiento y seguimiento del proceso hasta que el niño logre la readaptación al medio escolar después de finalizar su estancia en el hospital.

Más concretamente, autores como Ullán (2005) subrayan que las actividades a desarrollar en las aulas hospitalarias pueden ser de tres tipos:

- Curriculares o educativas, adaptando el currículo ordinario de los niños ingresados con el fin de continuar el proceso de enseñanza-aprendizaje dentro del hospital.

- Lúdico-formativas, para reforzar el desarrollo de la personalidad y favorecer la socialización con los nuevos compañeros del hospital.

- Actividades complementarias, dirigidas a mejorar la situación personal y psicoafectiva del niño hospitalizado.

En relación con las actividades lúdico-formativas, esa última autora acentúa que tales actividades deberían potenciarse en las aulas hospitalarias, dado su carácter creativo y participativo. Parte de la base de que el juego es un elemento crucial del desarrollo infantil, del que se derivan altas aportaciones para el bienestar psicológico, cognitivo, social y emocional de los niños, pues al jugar el menor se divierte, desarrolla recursos cognitivos y emocionales, aprende a resolver situaciones y a controlarse a sí mismo, su cuerpo y su entorno. En el caso de los alumnos hospitalizados, el juego se presenta como una herramienta fundamental para ayudarles a expresar sus emociones, proporcionarles sentimientos de dominio y de control, liberar sus angustias.

En cualquier caso, la clave estará en diseñar actividades adaptadas a las características y necesidades específicas de cada niño enfermo y/u hospitalizado, priorizando aquéllas que han de realizar en equipo, de cara a estrechar las relaciones entre ellos y evitar su aislamiento, afianzar su seguridad, hacerles sentir bien, relajarles ante su temor de ser rechazados por los demás compañeros, 
etc. Las emociones, en este caso las positivas, podrían acelerar, sin duda, su proceso de recuperación.

\section{Propuestas para la práctica psicodidáctica en centros hospitalarios}

Con el propósito de sensibilizar y abogar por una salida profesional de los psicopedagogos en entornos hospitalarios, se esbozan a continuación ejemplos de prácticas psicopedagógicas que se están llevando a cabo con pacientes pediátricos. Son actividades plausibles de desarrollar por los psicopedagogos en formación que cursen el Prácticum en hospitales, contribuyendo así a una preparación específica en el campo profesional de la Psicopedagogía Hospitalaria.

Para generar un ambiente dinámico, creativo y participativo, romper el aislamiento que produce el ingreso hospitalario, bloquear la aparición de pensamientos negativos y desdramatizar la enfermedad y olvidar el dolor, las Aulas Hospitalarias de la Comunidad de Castilla y León llevan a cabo, día tras día, diferentes actividades de carácter lúdico-formativas con los alumnos ingresados. Entre ellas, destacan las siguientes:

- Proyección de películas.

- Invitación de poetas, cuenta-cuentos, autores literarios, organizando charlas y coloquios enriquecedores con los niños.

- Excursiones por el hospital.

- Elaboración de un pequeño vídeo con saludos, dedicatorias de los niños a sus amigos, familiares, etc.

- Talleres de animación a la lectura y a la escritura.

- Juegos educativos clásicos: puzzles y juegos simbólicos: muñecos, marionetas ideales para el desarrollo de habilidades cognitivas y sociales.

- Juegos de participación social y de reglas: parchís, juegos de mesa.

- Y un sinfín de juegos de creatividad: dibujos, pinturas, manualidades con barro, plastilina, collages, recortables, caretas etc.; manifestaciones artísticas que se utilizan como elementos decorativos en el aula hospitalaria o en la propia planta de pediatría.

\section{Recursos y técnicas a emplear en el campo profesional}

"Es necesario que todos los encargados de la atención del niño, tanto en el hospital como en casa y la escuela, reconozcan la importancia de la actividad escolar y la fomenten con todos los recursos disponibles" (GÁNDARA; JUNQUERA, 2006, p. 16). A la hora de trabajar con el niño enfermo y/u hospitalizado se puede emplear una serie de técnicas encaminadas a fomentar su creatividad, el perfeccionamiento de sus destrezas, habilidades, capacidades de movimiento, limitaciones físicas y la utilización de nuevas tecnologías. Entre ellas, cabe destacar las siguientes:

a) La biblioteca del aula hospitalaria.

Siguiendo la experiencia desarrollada por Castillo (2006b) en el Aula Hospitalaria de León, estos profesionales pueden valerse de la biblioteca ubicada en el aula educativa del hospital para animar, por ejemplo, a los alumnos a que lean, para enseñarles a utilizar los diccionarios y las enciclopedias, para aprender a aprender, y para gozar con los libros de literatura infantil y juvenil que haya disponibles. Por las particulares características de estos alumnos (especial situación anímica, tiempo de estancia en el hospital), "el papel de una 'Biblioteca Viva' en la que los libros son puentes hacia el exterior, nos integran a distancia en nuestras clases, nos llevan a lugares fantásticos, y/o, en ocasiones, nos hacen olvidar" (CASTILLO, 2006b, p. 13).

b) Las nuevas tecnologías de la información y la comunicación.

Las nuevas tecnologías brindan múltiples posibilidades en el hospital para el trabajo con los niños enfermos. Así, por ejemplo, aprovechando los rincones de juego, pueden emplearse los ordenadores con internet, vídeos, DVDs, etc. En concreto, los profesionales del Aula Hospitalaria del Hospital Pío del Río 
Hortera de Valladolid se valen de algunos de estos recursos, entre ellos: juegos educativos como los de Pipo, Salto y Celia, Mía o Paula para que las tareas escolares sean más amenas y afianzar a la vez los conocimientos de los niños; programas como el PowerPoint o el Word para realizar esquemas de los temas de los alumnos de la Educación Secundaria Obligatoria (ESO) o realizar trabajos de síntesis; y Photoshop o Paint para que los alumnos jueguen con las imágenes y realicen composiciones originales o cambios imaginativos en fotos previamente realizadas en el aula hospitalaria.

Asimismo, encontramos el Programa Teleeducación en 32 Aulas Hospitalarias ${ }^{2}$, puesto a disposición de los niños enfermos, es uno de los proyectos más ambiciosos creados por el Ministerio de Educación y Cultura en 2005, a través del Programa de Nuevas Tecnologías. La idea se enmarca dentro de una política de innovación educativa, cuyo objetivo es aportar los nuevos medios telemáticos e informáticos como impulsores del desarrollo afectivo/social, la comunicación entre los alumnos ingresados y la recuperación del desfase académico. De este modo, se puede favorecer la coordinación con el centro educativo de referencia y la comunicación entre el alumnado, sin prescindir de la tarea psicopedagógica del docente.

c) Material bibliográfico.

Algunas de las obras literarias que estos profesionales pueden leer antes de su inmersión en las aulas hospitalarias, como medio de aproximación al conocimiento de la situación real que viven los niños enfermos hospitalizados, son: Conde (1997), Guibert (1992), Heredia (1998), Melvin y Perry (1991) y Tricker (1996).

\section{2. www.cnice.mecd.es/proyectos/aulashosp/proyecto.htm}

También se recomienda consultar algunos de los manuales específicos que sobre ese campo de actuación profesional existen en el mercado. Entre ellos: Costa (2000), Palomo (1995) y Valdés y Flórez (1995).

\section{Conclusiones de cara al futuro de la Psicopedagogía Hospitalaria}

Para concluir, conviene remarcar dos cuestiones fundamentales: la primera, si bien es cierto que el campo de atención de la Psicopedagogía Hospitalaria se sitúa preferentemente en torno al niño enfermo y/u hospitalizado, no menos lo es que, por extensión e implicación, éste debe incidir de manera especial en el campo de su familia y del personal sanitario, estableciéndose una estrecha colaboración entre el equipo interdisciplinario de profesionales que atienden al menor (médicos, enfermeros, asistentes sociales, animadores socioculturales etc.) y los familiares (LIZASOÁIN, 2003; OLIVARES, MÉNDEZ, ROS, 2002).

Y la segunda, surge al plantearnos una cuestión básica: ¿qué es lo más importante para lograr que los alumnos que se ven inmersos en un ambiente hospitalario mantengan su nivel de aprendizaje? Por lo expuesto en el presente artículo, la respuesta parece clara: que los profesionales que atiendan a estos alumnos posean una buena preparación, sobre todo por lo interdisciplinario y cooperativo de su trabajo (ORTIZ, 1999). Una respuesta que, sin duda, demanda más y mejores planes formativos de iniciación y perfeccionamiento del profesional de la educación (TONATTO; PROENÇA, 2007), en general, y de la Psicopedagogía Hospitalaria, en particular, organizados por las administraciones, centros de formación y recursos para el profesorado y, sobre todo, por las universidades españolas. 


\section{Referencias}

ALVES, Manuel; ORTIZ, María del Carmen; SERRADAS FONSECA, Marian. El método eduterapéutico como estrategia de apoyo al niño hospitalizado. Revista de Educación, Madrid, n. 335, p. 229-246, 2004.

BARCELÓ GALDÁCANO, Fermín; LAMARCA HURBE, Iñigo. Personas menores en situaciones de especial vulnerabilidad: retos para una intervención eficaz. Revista de Psicodidáctica, País Basco, n. 1, p. 25-35, 2006.

BARRUECO, Angel; ORTIZ, María del Carmen; CALVO, Isabel; DE MANUELES, Julio; MARTÍN, José. María. La acción educativa en la asistencia al niño hospitalizado. Revista de Educación Especial, Andalucía, v. 23, p. 39-54, 1997.

BRASLAVSKY, Cecília. Bases, orientaciones y criterios para el diseño de programas de formación de profesores. Revista Iberoamericana de Educación, Madrid, v. 19, p. 13-50, 1998.

CABALLERO, Solange Ana Maria. El aula hospitalaria: un camino a la educación inclusiva. Investigación Educativa, Murcia, v. 11, n. 19, p. 153-161, 2007.

CASTILLO, Maria Dolores. La coordinación en un aula hospitalaria. Revista de Aulas Hospitalarias, Castilla y León, v. 2, p. 16, 2005.

Escuela para todos: el aula hospitalaria del complejo hospitalario de León. In Actas de las XXIII Jornadas Nacionales de Universidades y Educación Especial "Hacia una educación sin exclusión”. CD-Rom. Murcia: Dpto. de Didáctica y Organización Escolar de la Universidad de Murcia, 2006a.

Biblioteca en el aula. Revista de Aulas Hospitalarias, Castilla y León, v. 3, p. 13, 2006b.

CONDE, J. L. Un caso de inocencia. Madrid: Debate, 1997.

COSTA, M. El juego y el juguete en la hospitalización infantil. Valencia: Nau Llibre, 2000.

DEASY-SPINETTA, Pat. School issues and the child with cancer. Cancer, New Jersey, v. 71, p. 3261-3264, 1993.

DE MANUELES, Julio, et. al. Necesidad de asistencia educativa al niño hospitalizado. Enseñanza: Anuario Interuniversitario de Didáctica, Salamanca, v. 20, p. 243-258, 2002.

DOVAL, María Isabel. Telemática y Pedagogía Hospitalaria. Estado de la cuestión. Revista Interuniversitaria de Tecnología Educativa, Oviedo, v. 0, p. 177-190, 2000.

GÁNDARA, Salomé; JUNQUERA, María Paz. Volver a la escuela. Revista de Aulas Hospitalarias, Castilla y León, v. 3, p. 16, 2006. GONZÁLEZ, Crescenciana. El Aula Hospitalaria del Hospital General Yague de Burgos. Revista de Aulas Hospitalarias, Castilla y León, v. 1,p. 8, 2004.

GONZÁLEZ-SIMANCAS, J. L.; POLAINO-LORENTE, A. Introducción a la Pedagogía Hospitalaria. In: GONZÁLEZ-SIMANCAS, J. L.; POLAINO-LORENTE, A. (eds.). Pedagogía Hospitalaria. Madrid: Narcea, 1990, p. 158-182.

GRAU, Cláudia. A pedagogía Hospitalaria. Revista Galega do Ensino, Galícia, n. 32,p. 169-182, 2001.

Necesidades educativas especiales derivadas de problemas crónicos de salud. In: GALLEGO, J. L.; FERNÁNDEZ, E.

(Coords.). Enciclopedia de educación infantil. Archidona, Málaga: Aljibe, 2003, p. 781-804.

GRAU, Claudia; ORTIZ, María del Carmen. La pedagogía hospitalaria en el marco de una educación inclusiva. Archidona, Málaga: Aljibe, 2001.

GUIBERT, Hervé. Al amigo que no me salvó la vida. Barcelona: Círculo de Lectores, 1992.

GUILLÉN, Manuel; MEJÍA, Angel. Actuaciones educativas en Aulas Hospitalarias. Atención escolar al niño enfermo. Madrid: Narcea, 2002. 
HEREDIA, Raquel. La agenda de los amigos muertos. Barcelona: Plaza \& Janés, 1998.

LEÓN, M. J. Educación especial para psicopedagogos. Granada: Grupo Editorial Universitario, 2001.

LIZASOÁIN, O. Educando al niño enfermo. Perspectivas de la pedagogía hospitalaria. Pamplona: Eunate, 2000.

. La pedagogía hospitalaria en una sociedad en cambio. In: OCHOA, B.; LIZASOÁlN, O. (Eds.). Intervención psicopedagógica en el desajuste del niño enfermo crónico hospitalizado. Pamplona: Universidad de Navarra-EUNSA, 2003, p. 78-99.

LÓPEZ, Isabel; FERNÁNDEZ, Antonio. Hospitalización infantil y atención psico-educativa en contextos excepcionales de aprendizaje. Revista de Educación, n. 341, Madrid, p. 553-577, 2006.

MELVIN, D. M.; PERRY, P. Más cerca de la luz. Madrid: Edad, 1991.

OLIVARES, J. F.; MÉNDEZ, F.X.; ROS, M. C. El entrenamiento de padres en contextos clínicos y de la salud. In: CABALLO, V.; SIMÓN, M. A. (Dir.). Manual de psicología clínica infantil y del adolescente: trastornos específicos. Madrid: Pirámide, 2002, p. 127-156.

ORTIZ, M. C. Aulas hospitalarias: aulas inclusivas. In: PÉREZ, R.; PASCUAL, M. A.; ÁLVAREZ, M. C. (Coords.). Educación y Diversidad. XV Jornadas Nacionales de Universidades y Educación Especial. Oviedo: Universidad de Oviedo, 1998, p. 285-298.

Formación de los profesionales del contexto hospitalario: profesorado. Revista de Currículum y Formación del Profesorado, Granada, v. 3, p. 105-120, 1999.

ORTIZ, María del Carmen; SERRADAS, Marian. Atención educativa al niño hospitalizado. In:Actas del Congreso Nacional de Educación: "La universidad en la formación del profesorado, una formación a debate". Burgos: Universidad de Burgos, 2002, p. 459.

PALOMO DEL BLANCO, María del Pilar. Análisis de un contexto social: Entorno hospitalario infantil e implicaciones educativas. Siglo Cero, Madrid, n. 141, p. 40-49, 1992.

. El niño hospitalizado: características, evaluación y tratamiento. Madrid: Pirámide, 1995.

ZIBETTI, Maria Lúcia Tonatto; SOUZA, Marilene Proença R. de. Apropriação e mobilização de saberes na prática pedagógica: contribuição para a formação de professores. Educação e Pesquisa, São Paulo, v. 33, n. 2, p. 247-262, maio/ago. 2007.

TRICKER, A. Voy a vivir: cuando parece que no hay salida. Madrid: [s. n.], 1996.

ULLÁN, Ana Maria. Proyecto curARTE de juego y creatividad en niños hospitalizados. Revista de Aulas Hospitalarias, Castilla y León, v. 2, p. 4-5, 2005.

VALDÉS, C. A.; FLÓREZ, J. A. El niño ante el hospital: programas para reducir la ansiedad hospitalaria. Oviedo: Universidad de Oviedo, 1995

Recebido em: 13.04.2010

Aprovado em: 12.09.2010

Francisco Javier Blanco Encomienda es Profesor de la Universidad de Granada y miembro del Grupo de Investigación Modelos Probabilísticos aplicados a las Ciencias Sociales.

María José Latorre Medina es Profesora de la Facultad de Educación y Humanidades de Ceuta de la Universidad de Granada y miembro del Grupo de Investigación Interuniversitario FORCE (Formación del Profesorado Centrada en la escuela). 